\title{
Integration of forest mapping and inventory to support forest management
}

\section{Corona P}

Forest inventory and forest mapping can be considered as monitoring and assessment applications that respond to different demands. However, the integration of mapping and inventory provides an effective framework for the support of forest management from multiple perspectives: (i) use of thematic maps for stratifying the inventory sample for the purpose of improving the precision of inventory estimates; (ii) coupling remotely sensed and sample inventory data for the purpose of constructing maps of inventoried forest attributes; (iii) coupling remotely sensed data and sample inventory data for the purpose of improving the precision of the inventory estimates; (iv) using inventory data as prior information to support thematic mapping; and $(v)$ using inventory data to correct map areal estimates. This paper aims to provide general considerations on this integration issue in the form of a scientific review and commentary discussion.

Keywords: Thematic mapping, Forest inventory, Monitoring and assessment programs, Remote sensing, Probability sampling

\section{Introduction}

Sound forest ecosystem management depends on accurate, complete, and concise information regarding the extent, condition and productivity of the natural resources. Es timation of these attributes is commonly an agreed objective of forest monitoring and assessment programs at a variety of spatial scales (Kleinn 2002, Corona \& Marchetti 2007). Each of such properties can also be investigated with regard to its change with time. Forest management information needs are focused and fostered by the global context, in relationship to biodiversity, soil and water conservation, timber provision, nonwood products, potential to sequester atmospheric carbon, etc. Distinctively, monitoring and assessment programs are valuable for providing broad overviews to help strategic and tactical planning development and allow to place forest managers into a perspective that facilitates the prioritization of limited financial resources usually available.

Technological advancements have led to

Dipartimento di Scienze dell'Ambiente Forestale e delle sue Risorse, Università della Tuscia, v. San Camillo de Lellis, I-01100 Viterbo (Italy)

\section{(a) Piermaria Corona}

(piermaria.corona@unitus.it)

Received: Dec 04, 2009 - Accepted: Jan 23, 2010

Citation: Corona P, 2010. Integration of forest mapping and inventory to support forest management. iForest 3: 59-64 [online: 2010-05-17] URL: http://www.sisef.it/ iforest/show.php?id=531 sophisticated tools for the acquisition of current, meaningful, and accurate information, and methodological developments have contributed to greater efficiency in the processing and management of such information (Köhl et al. 2006).

Forest inventory and forest mapping can be considered as monitoring and assessment applications that respond to different demands. For forest inventory, the demand is for information pertaining to the amount of forest resources and related attributes in a given region, and is satisfied by investigating a small part of the region in the form of a sample. For forest mapping, the demand is for a geographical depiction of the location of forest and related attributes within the region. These different objectives are targeted by different operational approaches which may vary considerably with the size of the surveyed region.

The integration of inventory and mapping data is emerging as a major issue for the development of programs that monitor and assess land and multiple environmental functions. Stehman (2009) highlights this issue in the context of estimating the area of land cover and land cover change. From this perspective, the present paper aims to provide general considerations on the integration of forest inventory and mapping in the form of commentary discussion for the community of foresters with basic knowledge of geomatics and inventory.

\section{Forest mapping}

For purposes of supporting operational forest management, the cartographic representation of forest cover is generally based on a minimum mapping unit defined to be 0.5 ha (Vidal et al. 2008). Thus, remotely sensed imagery with high and very high geometric resolution is required.

Remote sensing is defined as the acquisition of data from sensors on board aircraft or space-based platforms. There are two categories of sensors, passive and active: the first measures the reflectance of naturally occurring solar radiation (e.g., photography), and the second measures radiation that is transmitted from the sensor and reflected from the earth's surface back to the sensor (e.g., radar). Aircraft sensors principally involve aerial photos linked to a geographical reference system or light detection and ranging (LiDAR) imagery, with image resolutions of $1 \mathrm{~m}$ or less. Satellite-based sensors acquire images covering large geographical areas and have variable geometric resolution, ranging from submetric to kilometers (for further background on remote sensing, see Campbell 2007; for forestry application, see Wulder \& Franklin 2003).

Until recently, aerial imagery has been the main source of remotely sensed data for operational purposes. However, in the last two decades new technologies have enabled a transition from data such as a mosaic of photos to digital imagery in form of a matrix of pixels and has produced many advantages for data acquisition, management and analysis. Meaningful examples of airborne digital imagery include the ADS40 (Reulke 2001) and the ASPIS (Papale et al. 2008) systems. An example of a typical airborne application is the assessment of forest burnt areas (Corona et al. 2008) in environments where most fires are small (e.g., less than 10ha wide) as in Europe (http://effis.jrc.ec.europa.eu/about/technical-background/). Airborne imagery is usually less efficient in terms of the ratio of quality to cost than satellite images for the multitemporal monitoring and assessment of forest resources for large areas. Satellite imagery with metric or submetric resolution in the panchromatic channel (e.g., Formosat, IKONOS, QuickBird, OrbView-3, WorldView-2, Pleiades) is suitable for mapping at scales of 1:50001:10000, while Landsat TM, Spot HRV, Spot5 and Aster data probably represent the current best trade-off in terms of the qualitycost ratio for mapping at scales of 1:250001:50000.

On-screen image interpretation is commonly adopted for forest mapping at a professional level: panchromatic and/or natural colors and/or false color (eventually fused with the panchromatic channel) images are the base for the manual delineation and thematic classification of the delineated vectorial objects (polygons). However, the quality of products obtained with this procedure 
is dependent to some degree on interpreter subjectivity.

Automatic (unsupervised) and semiautomatic (supervised) methods of multispectral image classification have been developed to produce cheaper and more objective products. Supervised methods are based on ground-truthing, a term used extensively to describe the best available determination of the true thematic class at a specified location. With supervised classification, groundtruth data is acquired for a specified number of training pixels for each thematic class as a means of determining the spectral signature (distribution of the digital numbers, DN, across the spectral channels) typical of the class. The multispectral reflectance of the target pixels (i.e., the pixels to be classified) is compared with the spectral signatures of the classes so that each target pixel can be assigned (e.g., by discriminant analysis) to the thematic class whose signature is most similar to the pixel's multispectral reflectance. Unsupervised methods do not require the acquisition of training pixels. Instead, the classification is carried out by grouping (cluster analysis) the pixels on the basis of their similarity in terms of multispectral reflectance. The resulting groups are viewed as thematic classes and labelled a posteriori by the interpreter. If ground data have been acquired, labels could be assigned to groups on the basis of the most common class (or some other measure) of the ground data points included in the group (for further details, see e.g., Campbell 2007).

The previously described approaches are characterized as pixel-oriented because the classification is carried out on a per-pixel basis. In the last decade alternative techniques have been developed based on objectoriented classification (Benz et al. 2004) of polygons produced by image segmentation Image segmentation refers to partitioning an image into meaningful regions based on either homogeneity or heterogeneity criteria (see Haralick \& Shapiro 1992) and represents the interface between image pre-processing and image interpretation (object recognition). The advantage of this approach is that greater information content can be associated with the polygons than with single pixels; such content includes the geometry and hierarchy of the polygons and the spectral heterogeneity of the pixels in each polygon. The segmentation of digital imagery can be carried out (semi)automatically faster and more objectively (Baatz \& Schäpe 2000) than manual polygon delineation. Map products obtained using digital segmentation and object-oriented classification are more suitable to customer expectations and more similar to the conventional maps obtained by manual interpretation than those produced by the pixel-oriented approach.

LiDAR techniques, particularly aerial laser scanning, have tremendous potential for supporting operational forest management and represent the frontier of current research in this area. By measuring forest canopy height and eventually the width and depth of individual tree crowns, LiDAR data can be used effectively to represent the structure of forest stands and to estimate standing wood volume, biomass, etc. Considerable research is ongoing to establish reliable and feasible survey protocols for integrating LiDAR and forest inventory data (e.g., Peterson et al. 2007, McRoberts \& Tomppo 2007, Næsset \& Gobakken 2008, Corona \& Fattorini 2008). This issue will not be directly addressed in this paper.

\section{Forest inventory}

A forest inventory is the statistical description of the quantitative and qualitative attributes of the forest resources in a given region. Forest inventory information is generally reported for management and/or administrative units (e.g., district, province, country) and/or for thematic or resource classes (e.g., forest type, age). Forest inventories are currently evolving towards multipurpose resources surveys (Lund 1998, Corona \& Marchetti 2007) and are broadening their scope in two major directions (Kleinn 2002): (i) inclusion of additional variables that are not directly related to timber assessment and wood harvesting, such as biodiversity attributes; (ii) expansion of the target population to include non-traditional objects such as trees outside forests and urban forests.

Forest inventory could, in principle, be based on a complete census for which every tree in a given region is measured. However, this is usually impossible because of the time and/or costs associated with the large areas involved. Therefore, information is typically acquired using sampling methods for which only a proportion of the population (the sample) is inspected, and inferences regarding the whole population are based on this sample (Kangas \& Maltamo 2007). Multiple sampling strategies (e.g., Gregoire \& Valentine 2008, Mandallaz 2008) are associated with the wide variety of types of forest inventories (for a typology of forest inventories, see Köhl et al. 2006). However, all sample-based inventories over large areas share a common methodological feature: sample units are objectively selected by rigorous probabilistic rules as a means of guaranteeing the credibility of estimates (Olsen \& Schreuder 1997).

Traditionally, forest inventory data are analysed in the framework of design-based inference for which population values are regarded as fixed constants and the randomization distribution resulting from the sampling design is the basis of inference. In this framework, the bias and variance of an estimator of a population parameter are de- termined from the set of all possible samples (the sample space) and from the probability associated with each sample. Särndal et al. (1992), Gregoire (1998) and Fattorini (2001) provide extensive discussion of design-based inference and contrast it with model-based inference. Usually, forest inventories adopt sampling schemes in which a set of points is randomly selected from the study region in accordance with a spatial sampling design. Subsequently, plots of adequate radius are then established with centres at the selected points, and forest attributes are recorded for the plots (e.g., De Vries 1986, Schreuder et al. 1993, Fattorini et al. 2006). Ground data obtained from these plots are the type to which this paper refers.

\section{Relationships between forest inventory and mapping}

The potential to integrate multisource information is a key element of forest monitoring and assessment programs (Lund 1998, Köhl et al. 2006, McRoberts \& Tomppo 2007). Inter alia, the relationships between forest inventory and mapping can be framed in the perspective of:

- exploiting thematic maps for stratifying the inventory sample for purposes of improving the precision of inventory estimates;

- coupling the remotely sensed data for an entire region with sample inventory data to produce maps of the inventoried forest attributes;

- coupling the remotely sensed data with the sample inventory data to improve the precision of the inventory estimates;

- exploiting the inventory data as prior information to support thematic mapping;

- exploiting the inventory data for the correction of map areal estimates.

\section{Exploiting thematic maps for stratifying} the inventory sample

Stratified sampling consists of dividing the population into subpopulations, called strata, that are relatively homogeneous, and then sampling each stratum separately. In most situations, stratified probability sampling is likely to yield more precise population estimates (i.e., estimates with smaller standard errors) than non-stratified probability sampling with the same sample size.

The strata can be obtained directly as thematic classes or groups of classes from a thematic map; in this case, the map implicitly provides the complete coverage of auxiliary information to improve the precision of the inventory estimates. The operational procedure (map polygon stratification) includes five steps: (1) the proportion of the area of each stratum with respect to the area of the mapped region is calculated; (2) a specified number of sample units is allocated to each stratum, usually in proportion to the area of the stratum as determined from the 
map or possibly in proportional to stratum variances; (3) sample units are geolocated independently within each stratum according to a given probability sampling scheme; (4) statistical parameters (e.g., mean, total and their variances) are estimated for each stratum; and (5) stratum estimates are combined to obtain the overall estimates for the population. Practical examples can be found in Lund \& Thomas (1989) and Suárez et al. (2004).

Post-stratification is often applied too: it is not used to select the sample, but is instead used to assign plots to strata after the sample has been selected. However, the same stratified estimators are used for the analysis When an inventory uses permanent plots, there is no opportunity for stratified sampling; nevertheless, stratified estimation may still substantially improve the precision of estimates. The auxiliary information is the proportion of area in each of the post-strata constructed from the mapped classes. Examples of post-stratification can be found in McRoberts et al. $(2002,2005)$ and Nilsson et al. (2005).

Note that application of both the above approaches usually ignores complications arising from map errors and spatial misregistration of inventory data locations to the map: of course, the amount by which the stratification improves precision diminishes to the degree that such shortcomings are present. The issue of the above complications affects almost all the applications discussed in this paper but it will not be further addressed.

\section{Coupling remotely sensed data and in-} ventory data to map forest attributes

Estimation of the relationship between remotely sensed data and the biophysical attributes of forest vegetation (standing wood volume, biomass increment, etc.) permits maps of the attributes observed at the sample inventory units to be constructed for the entire region of interest, i.e., the attributes can be predicted for all the pixels in the region thus producing maps. The exploited auxiliary variables are usually the DNs of the spectral channels (and/or their combination to produce vegetation indices, e.g., Maselli et al. 2005) which are available for all the $N$ pixels in the region, while the values of the $Y$-variable of interest (the forest attribute) are known only for the sample of $n$ pixels corresponding to the inventory sample units, characterized as the reference set.

The mapping procedures can be based on either parametric or non-parametric approaches to predicting the values of $Y$ for pixels that do not correspond to the inventory sample units, characterized as the target set. Non-parametric approaches are distribution-free in that they do not rely on any underlying probability distribution for estima- tion.

Nearest Neighbors (NN) techniques are well known non-parametric approaches whose operational application is increasing, even at the forest professional level. Other non-parametric approaches such as decision trees (classification and regression tree, Random Forest) and neural networks (multilayer perceptrons, self-organising maps, radial basis function networks, adaptive resonance theory networks, etc.) are promising, albeit usually less effective than NN techniques for mapping forest attributes (e.g., McInerney \& Nieuwenhuis 2009, Stümer et al. 2010). NN techniques predict the unknown value of $Y$ for the $j$-th target pixel as a weighted mean of the $Y$ values for the $k$ reference pixels nearest to the $j$-th target pixel in the multidimensional space defined by the auxiliary variables (eqn. 1):

$$
\tilde{y}_{j}=\sum_{i=1}^{k} w_{i} y_{j i}
$$

where $k(<n)$ denotes the number of neighbours adopted for the prediction and the $w_{\mathrm{i}} \mathrm{S}$ are weights such that $w_{1}+\ldots+w_{\mathrm{i}}=1$. A straightforward and suitable choice for the weights is $w_{\mathrm{i}}=1 / k$ for any $i=1, \ldots, k$ (McRoberts et al. 2007, Baffetta et al. 2009), but they are often selected to be inversely proportional to the multidimensional distance between the $j$-th target pixel and each of the $k$ nearest neighbor reference pixels. Examples of NN applications are provided by Franco-Lopez et al. (2001), Tomppo et al. (2002a, 2009), Chirici et al. (2008), McRoberts (2009a).

Among parametric approaches, the most commonly used is generalized regression (GREG) for which the prediction of $Y$ for each target pixel is based on a regression (or ratio) established between $Y$ and the auxiliary variables using data from the reference set. The adopted model is often linear, such as (eqn. 2):

$$
Y=\beta_{0}+\beta_{1} X_{1}+\ldots+\beta_{q} X_{q}
$$

where $X_{1}, \ldots, X_{\mathrm{q}}$ are the auxiliary variables. However, the GREG estimator may encompass a wide range of additional models. Examples of application of the GREG approach are provided by Moisen \& Edwards (1999), Puhr \& Donoghue (2000) and Opsomer et al. (2007).

All other factors being equal, some Monte Carlo investigations empirically demonstrate that GREG is usually more effective than NN (Baffetta et al. 2009), but in some situations it may give rise to unlikely results (e.g., negative predictions). A major advantage of the NN approach is that it is multivariate in the sense that it can estimate multiple $Y$-variables simultaneously and still retain their complex variance-covariance structure and natural variation within the bounds of biological reality, at least as long as $k=1$ (see McRoberts 2009b). On the contrary, with regression approaches $Y$-variables are often estimated separately which may lead to estimates with unreasonable relationships and variance-covariance structures that differ greatly from the original field data (Eskelson et al. 2009). However, with respect to the above issues, $\mathrm{NN}$ tends to behave more like regression approaches as $k$ increases.

Coupling remotely sensed data with the sample inventory data to improve the precision of the inventory estimates

This section is complementary to the preceding one in that the maps constructed using auxiliary remotely sensed information that is correlated with the $Y$-variables of interest may also be used to more efficiently estimate statistical parameters (e.g., Nilsson et al. 2003).

In the case of model-assisted estimation, if the locations of the reference set $S$ (the inventory sample) are obtained using simple random or systematic sampling without replacement, then prediction approaches such as $k \mathrm{NN}$ and GREG can be used with the approximately unbiased estimator of the population total $Y$ over the entire study region given by (eqn. 3):

$$
\hat{T}_{\text {asst }}=\sum_{j=1}^{N} \tilde{y}_{j}+\frac{N}{n} \sum_{j \in S} e_{j}
$$

where $\tilde{y}_{\mathrm{j}}$ denotes the predicted value of $Y$ for the $j$-th pixel and $e_{\mathrm{j}}=y_{\mathrm{j}}-\tilde{y_{\mathrm{j}}}$ denotes the prediction error. Moreover, an approximately conservative estimator of the sampling variance is given by (eqn. 4):

$$
\hat{V}\left(\hat{T}_{\text {asst }}\right)=\frac{N(N-n)}{n(n-1)} \sum_{j \in S}\left(e_{j}-\bar{e}\right)^{2}
$$

where (eqn. 5):

$$
\bar{e}=\frac{1}{n} \sum_{j \in S} e_{j}
$$

If only the sample inventory data from the reference set are used for estimation (i.e., the auxiliary information from remote sensing is not used), then the unbiased estimator of the total $Y$ under simple random sampling without replacement is (eqn. 6):

$$
\hat{T}=\frac{N}{n} \sum_{j \in S} y_{j}
$$

while the unbiased estimator of the sampling variance is (eqn. 7):

$$
\hat{V}(\hat{T})=\frac{N(N-n)}{n(n-1)} \sum_{j \in S}\left(y_{j}-\bar{y}\right)^{2}
$$

where $\bar{y}=\hat{T} / N$.

If the relationship between $Y$ and the auxiliary variables is sufficiently strong, the model-assisted estimator $\hat{T}_{\text {asst }}$ tends to be more precise than $\hat{T}$. In relative terms, the relative advantage of $\hat{T}_{\text {asst }}$ over $\hat{T}$ with respect to precision increases as the size of the reference set decreases. Note, however, that 
even the estimation error of $\hat{T}_{\text {asst }}$ may be large for small areas with few sampled pixels. This problem is well-recognized in the statistical literature as small area estimation and can be handled only by using model-based approaches (e.g., Rao 2003).

Model-assisted estimators can be used with any probability sampling design (for general formulation of the estimators - see Baffetta et al. 2009, chapt. 3.2) and allow great flexibility in modelling the relationship between the $Y$ and the auxiliary variables. While the improvement in the precision of a model-assisted estimator is dependent on how well the specified model corresponds to the actual relationship between the $Y$ and the auxiliary variables, the validity of the inference is not dependent on correct model specification but instead remains based on the randomization distribution associated with the sampling design. In particular, model-assisted estimators are approximately (asymptotically) design unbiased regardless of whether the working model is correct or not, and are particularly efficient if the working model is correct.

Inventory data as prior information to support thematic mapping

The information from forest inventory data about the distribution of given thematic classes in relation to environmental characteristics can be exploited as a priori knowledge for the thematic classification of remotely sensed imagery.

Prior probability can be incorporated in classical discriminant analysis (Tomppo et al. 2002b) and, more generally, with Bayesian classifiers. The radiometric information in the remote sensing data (i.e., the multispectral reflectance of each pixel/polygon) is combined with the additional, independently available forest inventory data (the prior information) to produce a full probability distribution (posterior distribution), so that the class with the highest posterior probability can be assigned to each pixel or polygon. This approach exploits the potential of forest inventory data for establishing quantitative relationships between the spatial distribution of the thematic classes (e.g., forest types) to be mapped and environmental factors such as altitude, exposure, soil type, etc. Examples of forest mapping applications are provided by Shataee \& Darvishsefat 2004 and Finley et al. (2008).

\section{Exploiting inventory data for the correction of map areal estimates}

There are important risks in using thematic maps produced by interpretation of remotely sensed imagery as a direct tool to estimate spatial variables. When mapping, the interpretation errors tend to be systematic, and there is no compensation between commission and omission errors, i.e., areas of a land-use type A incorrectly mapped as landuse type B are not offset by areas of land-use type incorrectly mapped as A (Carfagna \& Gallego 1999, Corona 1999).

Area estimation can be viewed as a valueadded analysis appended to a forest inventory when the sample obtained from the inventory is used to estimate the area of each class from a given map, i.e., the confusion matrix obtained from the inventory sample is used to adjust the area of each thematic class (e.g., Stehman 2009, McRoberts 2009c). Obviously this is feasible only when the inventory nomenclature is analogous to that of the map.

If points are selected completely at random over the study area (simple random sampling) or randomly within the polygons partitioning the study area (tessellation stratified sampling) the classical estimator for the size of the area of type $m$ is (eqn. 8):

$$
\hat{A}_{m}=A \hat{p}_{m}
$$

where $\hat{p}_{\mathrm{m}}$ is the proportion of inventory points classified as type $m$ with respect to the total number of inventory points, say $n$, and $A$ is the size of the study region. In this case the variance estimator (eqn. 9):

$$
\hat{V}\left(\hat{A}_{m}\right)=A^{2} \cdot \frac{\hat{p}_{m}\left(1-\hat{p}_{m}\right)}{n-1}
$$

is unbiased under simple random sampling and conservative under tesselation stratified sampling (Fattorini et al. 2004). However, if a thematic map of the study area is previously available, and if $A_{1}, \ldots, A_{\mathrm{c}}$ are the areas of the $C$ thematic classes partitioning the map, an alternative estimator for the area of type $m$ is given by (eqn. 10):

$$
\hat{A}_{m}^{*}=\sum_{h=1}^{C} A_{h} \hat{p}_{h m}
$$

where $\hat{p}_{\mathrm{hm}}$ represents the proportion of the inventory points mapped as forest type $h$ but actually belonging to forest type $m$. In this case the variance estimator (eqn. 11):

$\hat{V}\left(\hat{A}_{m}^{*}\right)=\sum_{h=1}^{C} A_{h}{ }^{2} \frac{\hat{p}_{h m}\left(1-\hat{p}_{h m}\right)}{n-1}-2 \sum_{l>h=1}^{C} A_{l} A_{h} \frac{\hat{p}_{h m} \hat{p}_{l m}}{n-1}$

is once again unbiased under simple random sampling and conservative under tesselation stratified sampling.

Gallego (2004) provides a comprehensive review of area estimation methods, Stehman \& Foody (2009) review basic methods of accuracy assessment, and Stehman (2009) uses model-assisted estimation as a unifying framework for estimating the area of land cover and land-cover change from remote sensing.

For the sake of completeness, it must be acknowledged that even model-based approaches are gaining importance for this topic area. The criteria underlying modelbased inference differ considerably from those underlying the design-based inference for the above mentioned model-assisted estimation framework. The statistical properties of design-based estimators are derived with respect to all the possible samples arising from the adopted sampling scheme, considering the population values as a set of fixed constants. On the other hand, the properties of model-based estimators are obtained with respect to all the populations which may be generated from the assumed superpopulation model, considering the sample as fixed (i.e., purposively selected). The validity of a model-based inference is based on the validity of the model, not the probabilistic nature of the sample as is the case for design-based inference. In fact, purposive, non-probability samples may produce entirely valid model-based inferences. Model-based approaches are distinctively (but not only) suitable for small areas, as already stressed (see § 4.3). A model-based approach to estimating forest area is reported by McRoberts (2006).

\section{Final remark}

The improvement of forest surveys through multi-purpose and multi-source networks is a topic of increasing interest and is usually regarded more positively by the stakeholders, governmental or not, than establishment of new monitoring and assessment programs. Support for forest management should therefore be framed according to a multi-faceted approach that integrates mapping and inventory as a means of providing comprehensive knowledge on the state and trends of forest resources as well as on the interactions and interdependencies with other land uses.

As remote sensing technology and associated analytical methods continue to improve rapidly with reasonable costs, they are likely to play an even more substantial role for forest monitoring and assessment in the future. In this light, it must be stressed that the conceptual and methodological differences between forest mapping and forest inventory are often unduly amplified by the lack of standardization / harmonization between their nomenclature systems. Instead, nomenclature systems may constitute fundamental bridges, and the value of shared and integrated typological frameworks, from continental (e.g., Barbati et al. 2007) to local (e.g., Corona et al. 2004) scales, should be more readily acknowledged.

\section{Acknowledgements}

This paper was partially carried out under the project PRIN2007 "Innovative methods for the identification, characterization and management of old-growth forests in the Mediterranean environment" (research unit: DISAFRI, University of Tuscia; national coordinator: G. Chirici) funded by the Italian Ministry of University and Research. I would like acknowledging Fattorini L (Uni- 
versity of Siena, Italy), McRoberts RE (Forest Service, USDA, USA) and Tabacchi $\mathrm{G}$ (Italy) for the helpful discussions and precious suggestions.

\section{References}

Baatz M, Schäpe A (2000). Multiresolution segmentation: an optimization approach for high quality multi-scale image segmentation. In: "Angewandte geographische informationsverarbeitung XI beiträge zum AGIT-Symposium Salzburg" (Strobl J, Blascke T, Greisebener G eds). Herbert Wichmann Verlag, Karlsruhe, Germany.

Baffetta F, Fattorini L, Franceschi S, Corona P (2009). Design-based approach to k-nearest neighbours technique for coupling field and remotely sensed data in forest surveys. Remote Sensing of Environment 113: 463-475. - doi: 10.1016/j.rse.2008.06.014

Benz UC, Hofmann P, Willhauck G, Lingenfelder I, Heynen M (2004). Multi-resolution, object-oriented fuzzy analysis of remote sensing data for GIS-ready information. ISPRS Journal of Photogrammetry \& Remote Sensing 58: 239-258. doi: 10.1016/j.isprsjprs.2003.10.002

Barbati A, Corona P, Marchetti M (2007). A forest typology for monitoring sustainable forest management: the case of European forest types. Plant Biosystems 1: 93-103. - doi: 10.1080/ 11263500601153842

Campbell J (2007). Introduction to remote sensing. Fourth Edition. The Guildford Press, New York, USA.

Carfagna E, Gallego FJ (1999). Thematic maps and statistics. In: "Land cover and land use information systems for European Union policy needs". Office for Official Publications of the European Communities, Luxembourg, pp. 219228.

Chirici G, Barbati A, Corona P, Marchetti M, Travaglini D, Maselli F, Bertini R (2008). Nonparametric and parametric methods using satellite images for estimating growing stock volume in alpine and Mediterranean forest ecosystems. Remote Sensing of Environment 112: 26862700. - doi: 10.1016/j.rse.2008.01.002

Corona P (1999). Valutazione dell'accuratezza tematica in cartografia forestale. L'Italia Forestale e Montana 3: 153-161.

Corona P, Del Favero R, Marchetti M (2004). Stand-level forest type approach in Italy: experiences from the last twenty years. In: "Monitoring and indicators for Forest Biodiversity in Europe from ideas to Operationality" (Marchetti M ed). EFI Proceedings 51: 143-152.

Corona P, Marchetti M (2007). Outlining multi-purpose forest inventories to assess the ecosystem approach in forestry. Plant Biosystems 141 (2): 243-251. - doi: 10.1080/ 11263500701401836

Corona P, Lamonaca A, Chirici G (2008). Remote sensing support for post fire forest management. iForest 1: 6-12. - doi: 10.3832/ifor0305-0010006 Corona P, Fattorini L (2008). Area-based lidar-assisted estimation of forest standing volume.
Canadian Journal of Forest Research 38: 29112916. - doi: 10.1139/X08-122

De Vries PG (1986). Sampling theory for forest inventories. Springer-Verlag, New York, USA.

Eskelson BNI, Temesgen H, Lemay V, Barrett TM, Crookston NL, Hudak AT (2009). The roles of nearest neighbour methods in imputing missing data in forest inventory and monitoring databases. Scandinavian Journal of Forest Research 24: 235-246. - doi: 10.1080/02827580902870490 Fattorini L (2001). Design-based and model-based inference in forest inventories. ISAFA Comunicazioni di Ricerca 2: 13-23.

Fattorini L, Marcheselli M, Pisani C (2004). Twophase estimation of coverages with second-phase corrections. Environmetrics 15: 357-368. - doi: 10.1002/env.647

Fattorini L, Marcheselli M, Pisani C (2006). A three-phase sampling strategy for large-scale multiresource forest inventories. Journal of Agricultural, Biological and Environmental Statistics 11: 296-316. - doi: 10.1198/108571106X130548 Finley AO, Banerjee S, McRoberts RE (2008). A Bayesian approach to multi-source forest area estimation. Environmental and Ecological Statistics 15: 241-258. - doi: 10.1007/s10651-007-0049-5

Franco-Lopez H, Ek AR, Bauer ME (2001). Estimation and mapping of forest stand density, volume, and cover type using the k-nearest neighbours method. Remote Sensing of Environment 77: 251-274. - doi: 10.1016/S00344257(01)00209-7

Gallego FJ (2004). Remote sensing and land cover area estimation. International Journal of Remote Sensing 25: 3019-3047. - doi: 10.1080/ 01431160310001619607

Gregoire TG (1998). Design-based and modelbased inference in survey sampling: appreciating the difference. Canadian Journal of Forest Research 28: 1429-1447. - doi: 10.1139/cjfr-28-101429

Gregoire TG, Valentine HT (2008). Sampling strategies for natural resources and the environment. Chapman \& Hall, New York, USA.

Haralick RM, Shapiro LG (1992). Computer and robot vision. Addison-Weasley, Reading, UK.

Kangas A, Maltamo M (2007). Forest inventory. Methodology and applications. Springer, Dordrecht, The Netherlands.

Kleinn C (2002). New technologies and methodologies for national forest inventories. Unasylva 53: 10-15.

Köhl M, Magnussen S, Marchetti M (2006). Sampling methods, remote Sensing and GIS multiresource forest inventory. Tropical Forestry Series, Springer Verlag, Berlin-Heidelberg, Germany. - doi: 10.1007/978-3-540-32572-7

Lund G, Thomas CE (1989). A primer on stand and forest inventory designs. USDA Forest Service, General Technical Report WO-54, Portland, USA.

Lund G (1998). A comparison of multipurpose resource inventories (MRIs) throughout the world. EFI Working Paper 14, Joensuu, Finland.

Mandallaz D (2008). Sampling techniques for forest inventories. Chapman \& Hall, New York,
USA.

Maselli F, Chirici G, Bottai L, Corona P, Marchetti M (2005). Estimation of Mediterranean forest attributes by the application of k-NN procedure to multitemporal Landsat ETM+ images. International Journal of Remote Sensing 17: 3781-3796. - doi: 10.1080/01431160500166433 McInerney DO, Nieuwenhuis M (2009). A comparative analysis of $\mathrm{kNN}$ and decision tree methods for the Irish National Forest Inventory. International Journal of Remote Sensing 19: 49374955. - doi: 10.1080/01431160903022936

McRoberts RE (2006). A model-based approach to estimating forest area. Remote Sensing of Environment 103: 56-66. - doi: 10.1016/j.rse.2006. 03.005

McRoberts RE (2009a). A two-step nearest neighbours algorithm using satellite imagery for predicting forest structure within species composition classes. Remote Sensing of Environment 113: 532-545. - doi: 10.1016/j.rse.2008.10.001 McRoberts RE (2009b). Diagnostic tools for nearest neighbors techniques when used with satellite imagery. Remote Sensing of Environment 113: 489-499. - doi: 10.1016/j.rse.2008.06. 015

McRoberts RE (2009c). Satellite image-based estimates of forest area for international reporting. In: Proceedings of the "International Symposium on National Forest Inventory", Koresa Forest Research Institute, Korean Society of Forest Resources Measurements, Yangyang, South Korea, pp. 3-12.

McRoberts RE, Wendt DG, Nelson MD, Hansen MH (2002). Using a land cover classification based on satellite imagery to improve the precision of forest inventory area estimates. Remote Sensing of Environment 81: 36-44. - doi: 10.1016/S0034-4257(01)00330-3

McRoberts RE, Holden GR, Nelson MD, Liknes GC, Gormanson DD (2005). Using satellite imagery as ancillary data for increasing the precision of estimates for the Forest Inventory and Analysis program of the USDA Forest Service. Canadian Journal of Forest Research 35: 29682980. - doi: $10.1139 / \mathrm{x} 05-222$

McRoberts RE, Tomppo EO (2007). Remote sensing support for national forest inventories. Remote Sensing of Environment 110: 412-419. doi: 10.1016/j.rse.2006.09.034

McRoberts RE, Tomppo EO, Finley AO, Heikkinen JH (2007). Estimating areal means and variances of forest attributes using the k-nearest neighbours technique and satellite imagery. Remote Sensing of Environment 111: 466-480. doi: 10.1016/j.rse.2007.04.002

Moisen GG, Edwards TC (1999). Use of generalized linear models and digital data in a forest inventory of northern Utah. Journal of Agricultural, Biological and Environmental Statistics 4: 372-390. - doi: 10.2307/1400496

Næsset E, Gobakken T (2008). Estimation of above- and below-ground biomass across regions of the boreal forest zone using airborne laser. Remote Sensing of Environment 112: 3079-3090. doi: 10.1016/j.rse.2008.03.004 
Nilsson M, Folving S, Kennedy P, Puumalainen J, Chirici G, Corona P, Marchetti M, Olsson H, Ricotta C, Ringvall A, Stahl G, Tomppo E (2003). Combining remote sensing and field data for deriving unbiased estimates of forest parameters over large regions. In: "Advances in forest inventory for sustainable forest management and biodiversity monitoring" (Corona $\mathrm{P}$, Koehl M, Marchetti M eds). Kluwer, Dordrecht, The Netherlands, pp. 19-32.

Nilsson M, Holm S, Reese H, Wallerman J, Engberg J (2005). Improved forest statistics from the Swedish National Inventory by combining and optical satellite data using post-stratification. ForestSat 2005, Boras, Sweden, vol. 8a, pp. 2226.

Olsen AR, Schreuder HT (1997). Perspectives on large-scale natural resource surveys when causeeffect is a potential issue. Environmental and Ecological Statistics 4: 167-180. - doi: 10.1023/ A:1018522428238

Opsomer JD, Breidt FJ, Moisen GG, Kauermann G (2007). Model-assisted estimation of forest resources with generalized additive models. Journal of the American Statistical Association 102: 400-416. - doi: 10.1198/016214506000001491

Papale D, Belli C, Gioli G, Miglietta F, Ronchi C, Vaccari FP, Valentini R (2008). ASPIS, a flexible multispectral system for airborne remote sensing environmental applications. Sensors 8: 3240-3256. - doi: 10.3390/s8053240

Peterson B, Dubayah R, Hyde P, Hofton M, Blair JB, Fites-Kaufman JA (2007). Use of LIDAR for forest inventory and forest management application. In: Proceedings of the "Seventh annual forest inventory and analysis symposium", Portland, ME (McRoberts RE, Reams GA, Van Deusen PC, McWilliams WH eds). Gen. Tech.
Rep. WO-77, USDA, Forest Service, Washington, DC, USA, pp. 193-202.

Puhr CB, Donoghue DNM (2000). Remote sensing of upland conifer plantations using Landsat TM data: a case study from Galloway, south-west Scotland. International Journal of Remote Sensing 21: 633-646. - doi: 10.1080/ 014311600210470

Rao JNK (2003). Small area estimation. Wiley, New York, USA. - doi: 10.1002/0471722189 Reulke R (2001). Recent progress in digital photogrammetric stereo cameras and data evaluation. Springer, Berlin/Heidelberg, Germany.

Särndal CE, Swensson B, Wretman J (1992). Model assisted survey sampling. Springer-Verlag, New York, USA.

Schreuder HT, Gregoire TG, Wood GB (1993). Sampling methods for multiresource forest inventory. Wiley, New York, USA.

Shataee S, Darvishsefat AA (2004). Forest type classification improvement using spatial predictive distribution models. In: Proceedings of the " $21^{\text {st }}$ International Symposium on Remote Sensing of Environment”. St Petersburg (Russia). [online] URL: http://www.isprs.org/publications/ related/ISRSE/html/papers/927.pdf

Stehman SV (2009). Model-assisted estimation as a unifying framework for estimating the area of land cover and land-cover change from remote sensing. Remote Sensing of Environment 113: 2455-2462. - doi: 10.1016/j.rse.2009.07.006

Stehman SV, Foody GM (2009). Accuracy assessment. In: "The SAGE handbook of remote sensing" (Warner TA, Nellis MD, Foody GM eds). Sage Publications, New York, USA, pp. 297309.

Stümer W, Kenter B, Köhl M (2010). Spatial interpolation of in-situ data by self-organizing map algorithms (neural networks) for the assessment of carbon stocks in European forests. Forest Ecology and Management (in the press). - doi 10.1016/j.foreco.2010.04.008

Suárez MDR, Cruz L, Pérez Alegría LR (2004). A methodology for forest inventory using GIS techniques for carbon sequestration analysis in the Río Grande de Arecibo watershed. American Society of Agricultural and Biological Engineers, Annual Meeting, Paper no. 045003, St. Joseph, Michigan, USA.

Tomppo E, Nilsson M, Rosengren M, Aalto P, Kennedy P (2002a). Simultaneous use of Landsat-TM and IRS-1C WiFS data in estimating large area tree stem volume and aboveground biomass. Remote Sensing of Environment 82: 156-171. doi: 10.1016/S0034-4257(02)00031-7

Tomppo E, Czaplewski RL, Mäkisara K (2002b). The role of remote sensing in global forest assessment. A remote sensing background paper for Kotka IV expert consultation. Kotka, FAO, Rome.

Tomppo EO, Gagliano C, De Natale F; Katila M, McRoberts RE (2009). Predicting categorical forest variables using an improved k-Nearest Neighbour estimator and Landsat imagery. Remote Sensing of Environment 113: 500-517. doi: 10.1016/j.rse.2008.05.021

Vidal C, Lanz A, Tomppo E, Schadauer K, Gschwantner T, di Cosmo L, Robert N (2008). Establishing forest inventory reference definitions for forest and growing stock: a study towards common reporting. Sylva Fennica 42: $247-$ 266.

Wulder MA, Franklin SE (2003). Remote sensing of forest environments: concepts and case studies. Kluwer, Dordrecht, The Netherlands. 